triglycerides, total cholesterol, high density lipoprotein (HDL) cholesterol, apolipoproteins (AI and B), lipoprotein(a) and polymorphisms the G279A locus of the CETP gene, were determined.

Results In children with genotype B1B1, a significantly lower \% cholesterol concentration in the HDL fraction was found than in those with genotype B1B2 and B2B2. Children with genotype B1B1 were significantly shorter in stature than children with genotypes B1B2 and B2B2. Other biochemical parameters, such as levels of apolipoproteins (AI and B) and lipoprotein(a) were not significantly different between these genetic polymorphisms.

Conclusion In the future, proper early prevention based on the modification of risk factors, periodic lipid profile control, and the assay of markers of early atherosclerosis changes can reduce morbidity and mortality due to cardiovascular system diseases among children.

\section{DENVER II DEVELOPMENTAL TEST PERFORMANCE OF THE CHILDREN WITH IRON- DEFICIENCY ANEMIA BEFORE AND AFTER THE TREATMENT}

doi:10.1136/archdischild-2012-302724.0745

M Inalhan, T Ozlem, A Ozlem, C Mehmet, Y Feyza, Y Ayșegül. Clinics of Paediatrics, Zeynep Kamil Obstetrics, Gynecology and Paediatrics Training and Research Hospital, Istanbul, Turkey

Objective It is known that non-treatment of iron deficiency anemia in early childhood results mental and cognitive retardation. In our study we aimed to examine the Denver-II developmental test performance of the children with iron-deficiency or iron- deficiency anemia before and after 3 months of the treatment.

Materials and Methods 53 children aged between 1 and 6 years whom diagnosed as iron deficent or iron deficiency anemia were studied. Denver-II test were applied to the cases before and after 3 months of the treatment. The Denver Test results were classified as normal, suspecious and abnormal.

Results Suspicious Denver-II test results were found significantly higher in iron deficient anemic infants compared with iron deficient children $(p<0.01)$. The initial measurement level of ferritin and tranferrin saturation were found significantly low in children with suspicious test results compared with children with normal test results.

Before iron therapy, the rate of normal Denver test results in iron deficient anemic children was $42.5 \%$ and $81.5 \%$ in iron deficent children. After treatment the rate is $100 \%$ for iron deficient anemic children and $92.6 \%$ for iron deficient children.

Conclusion If iron deficiency could be treated before it becomes chronic or serious, motor, cognitive and behavioural development deficits can be prevented.

\section{GLAZMANN THROMBASTHENIA: SINGLE CENTER EXPERIENCE}

doi:10.1136/archdischild-2012-302724.0746

B Kutlubay, N Ozdemir, G Tuysuz, H Apak, T Celkan. Pediatric Hematology-Oncology Dept., Istanbul University, Cerrahpasa Medical Faculty, Istanbul, Turkey

Aim Glazmann thrombasthenia is a rare autosomal recessive disease characterized by defect in platelet aggregation. Here we report the management of children with Glazmann thrombasthenia followed at Cerrahpasa Medical Faculty Pediatric Hematology Department.

Methods Nineteen patients' (42\% girls, $58 \%$ boys; median age: 10 months) files were retrospectively reviewed.

Results The median age of the start of bleeding symptoms was 9 months (2 weeks-24 months). All patients presented with easy bruising and mucosal bleeding. Fourteen patients' parents were consanguineous. In 15 patients, flow cytometry was performed. According to this, 7 had type I, 6 had type II and 2 had type III disease. Nine patients were treated by thrombocyte transfusion, tranexamic acid, recombinant active factor VII and fibrin glue as a single or combined therapy in invasive procedures; none of them had a major bleeding complication.

Conclusion Bleeding control of invasive procedures may be challenging in children with Glazmann thrombasthenia; local treatments, DDAVP, steroid and antifibrinolytics may be used with success.

\section{ETHIOLOGICAL FACTORS AND PREVALENCE OF ZINC DEFICIENCY IN CHILDREN WITH IRON DEFICIENCY ANEMIA}

doi:10.1136/archdischild-2012-302724.0747

M Inalhan, Y Feyza, T Ozlem, A Ozlem, C Mehmet, 0 şuera. Clinics of Paediatrics, Zeynep Kamil Obstetrics, Gynecology and Paediatrics Training Research Hospital, Istanbul, Turkey

Introduction and aim: Iron deficiency is the most common nutritional deficiency in developing and developed countries. In the developing countries iron and zinc deficiency are seen together. In our study we researched the prevalence of zinc deficiency and etiological factors in iron deficiency anemia.

Materials and method A study group consisted of 40 children, aged between 1-14 years, diagnosed with iron deficiency anemia who submitted to our clinic with different complaints between January and August 2010. Control group consisted of 40 healthy childiren Age, sex, birth weights, history of mother's prophylactic iron suplement used in pregnancy, prophylactic iron supplement usage, breastfeeding duration time, initial supplement feeding age, meat, fruits-vegetables consumption, pica history, the blood count variables, serum iron levels, iron binding capacity, ferritin and serum zinc levels were recorded.

Findigs There was no significant difference between test and control groups in duration of breast feeding time and initial time for supplementary feeding $(p>0.05)$. Positive correlation was found between breast feeding time and iron levels and transferrin saturation indices $(p<0.01)$. Fruits and vegetables consumption were found significantly low in test grup.

Number of hospital admissions were also found to be significantly high in the test group.

The serum zinc levels of the children who have had prophylactic iron suplements were significantly high compared with the children who have had no prophylaxis $(\mathrm{p}<0.05)$.

Conclusions As a result of this research, factors namely breast feeding duration time, prophylactic use of iron supplements and fruit and vegetables consumptions have a large role to prevent children from the iron deficiency.

\section{DETECTION OF INTRON 22 GENE INVERSION (COMMON MUTATION) IN SEVERE HEMOPHILIA A IN KHOUZESTAN PROVINCE}

doi:10.1136/archdischild-2012-302724.0748

A Ghasemi, B Keikhaei, K Zandian, H Galedari. Pediatric Hematology-Oncology, Mashhad University of Medical Sciences, Mashhad, Iran

Introduction Hemophilia A, is the most common severe coagulation disorder with an incidence of 1 in 5000 males. The most common genetic alteration is a gene inversion, which occurs in approximately $45 \%$ of patients with severe hemophilia A.

The goal of the study was to assess the presence of the intron 22 inversion in patients with severe hemophilia A from Khozestan province.

Materials Twenty-five patients with severe hemophilia A and 15 mothers of these patients participated in the study patients were referred from the thalassemia service of Shafa Hospital to detect the 